\title{
Eigen-equation of Electronic Energy in Quantum Dot
}

\author{
Yu Lun Wu ${ }^{1}$, Shi Meng Feng, ${ }^{2, *}$ \\ ${ }^{1}$ School of Mechanical Engineering, Shanghai Jiao Tong University, China \\ ${ }^{2}$ Department of Physics and Astronomy, Shanghai Jiao Tong University, China
}

Copyright $\bigcirc 2016$ by authors, all rights reserved. Authors agree that this article remains permanently open access under the terms of the Creative Commons Attribution License 4.0 International License

\begin{abstract}
In this paper, we present one simple model of quantum dot to describe the potential. Based on the boundary continuity of wave function and its derivative, using the Chebyshev polynomial of the second kind and matrix theory, we deduced one eigen-equation of electronic energy which can clearly describe the relationship between the energy level and the surface potential in quantum dot. The further study shows that the eigen-equation of electronic energy is different when the material of quantum dot is different.
\end{abstract}

Keywords Quantum Dot, Eigen-equation, Electronic Energy

\section{PACS 73.21.La 78.67.Hc.}

\section{Introduction}

Semiconductor quantum dot is composed of a small number of atoms. The number of atoms is usually about a few to hundreds of atoms, and the size of the three dimensions is less than $100 \mathrm{~nm}$. In the three dimensions of the quantum dot, because the motion of the carrier is limited by the size effect and the quantum effect is very significant. Because of the special energy, the quantum dot exhibits the unique physical properties, such as quantum size effect and quantum tunneling effect, et, al. it has very important significance in the research of basic physics and new electronic and optoelectronic devices. Now a large number of studies have been reported on the energy level of the quantum dot [1-13]. For example, on the base of the adiabatic approximation, the adiabatic approximation with averaging and full numerical solution, Yiming Li, Jinn-Liang Liu et al solved the three dimensional Schrödinger equation, and gave qualitative as well as quantitative trends in electronic properties with various parameters[14].By the method of integrating directly the Schrödinger equation, Xiao-Yan Gu[15] gave the calculated energy spectra for two electrons in quantum dot given. $\mathrm{K} \mathrm{G}$ Dvoyan[16] used perturbation theory and limiting potential to study the energy states of electron in ellipsoidal quantum dot. Analytical expressions for particle energy spectrum have been obtained taking into account that electron effective masses are different in medium and in quantum dot. However, few reports completely describe the relationship of energy band or band gap with the surface potential, the interior periodic potential and the structure parameters.

In this paper, we try to study the dependence of the electronic energy on the quantum surface potential and other structure parameters. Our aim is to deduce an eigen-equation theoretical for describing the relationship of electronic energy with the surface potential, the internal periodic potential and structure parameters, which will be used to calculate the electronic energy in quantum dot.

\section{Theory}

In this paper, one simple model is presented (shown Figure.1). In the model, there is the periodic potential of $U_{0}$ when $0 \leq x \leq l=2 \mathrm{Nb}$ and the surface potential of $U_{1}$ when $x \leq 0$, or $x \geq l$

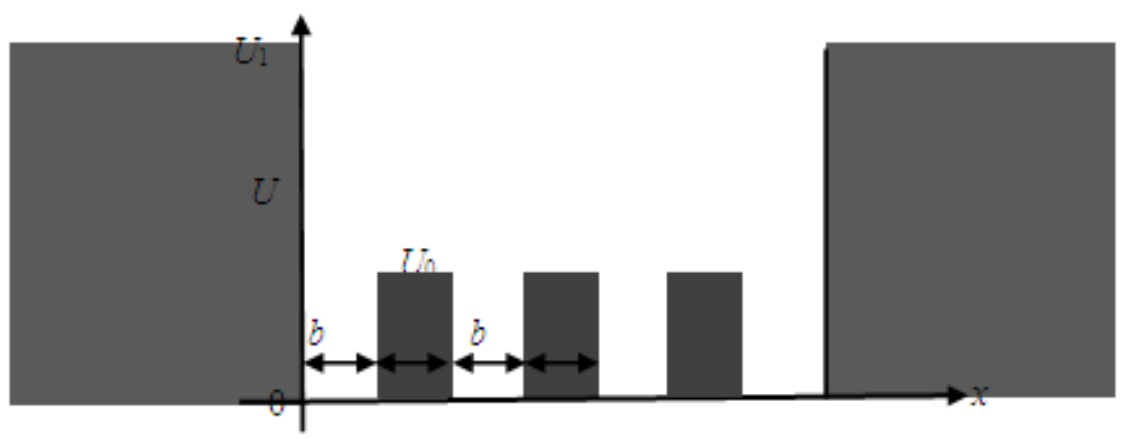

Figure 1. the simplified model of periodic potential in quantum dot 
Based on this model in Fig.1, Schrödinger equation:

$$
\left(-\frac{\hbar^{2} d^{2}}{2 m d x^{2}}+U\right) \psi(x)=E \psi(x)
$$

here $U(x)$ is the potential, $\psi(x)$ is the wave function, $E$ is the energy, $m$ is the electronic mass, $\hbar$ is a Plank constant. By solving one-dimensional Schrödinger equation, we can get, respectively

$$
\left\{\begin{array}{lcc}
\psi(x)=F_{0} e^{\beta_{0} x} & U=U_{1} & x \leq 0 \\
\psi(x)=A_{0} e^{i \alpha x}+B_{0} e^{-i \alpha x} & U=0 & 0 \leq x \leq b \\
\psi(x)=C_{0} e^{i \beta x}+D_{0} e^{-i \beta x} & U=U_{0} & b \leq x \leq 2 b \\
\psi(x)=A_{1} e^{i \alpha x}+B_{1} e^{-i \alpha x} & U=0 & 2 b \leq x \leq 3 b \\
\psi(x)=A_{N} e^{-\beta_{0}(x-2 N b)} & U=U_{1} & x \geq 2 N b
\end{array}\right.
$$

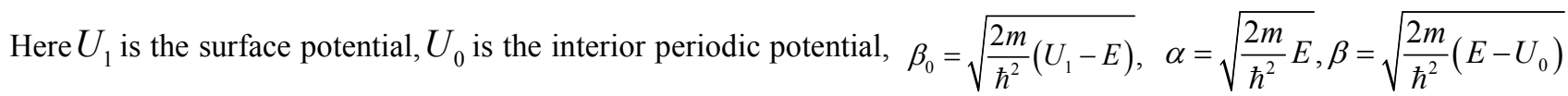
and $F_{0}, A_{0}, B_{0}, C_{0}, D_{0}, A_{1}, B_{1}$ are constant, respectively.

For the above model with the atom layer of $2 N+1$ in Fig.1, Using the method in the paper [22], we have

$$
\left[\begin{array}{l}
\psi(0) \\
\psi^{\prime}(0)
\end{array}\right]=\left\{\left[\begin{array}{cc}
\cos \alpha b & -\frac{1}{\alpha} \sin \alpha b \\
\alpha \sin \alpha b & \cos \alpha b
\end{array}\right]\left[\begin{array}{cc}
\cos \beta b & -\frac{1}{\beta} \sin \beta b \\
\beta \sin \beta b & \cos \beta b
\end{array}\right]\right\}^{N}\left[\begin{array}{cc}
\cos \alpha b & -\frac{1}{\alpha} \sin \alpha b \\
\alpha \sin \alpha b & \cos \alpha b
\end{array}\right]\left[\begin{array}{l}
\psi((N b+b)) \\
\psi^{\prime}((N b+b))
\end{array}\right]
$$

Based on the property of the transfer matrix, (3) can be changed into

$$
\begin{gathered}
{\left[\begin{array}{c}
\psi(0) \\
\psi^{\prime}(0)
\end{array}\right]=\left[\begin{array}{cc}
m_{11} U_{N-1}(\chi)-U_{N-2}(\chi) & m_{12} U_{N-1}(\chi) \\
m_{12} U_{N-1}(\chi) & m_{22} U_{N-1}(\chi)-U_{N-2}(\chi)
\end{array}\right]\left[\begin{array}{cc}
\cos \alpha b & -\frac{1}{\alpha} \sin \alpha b \\
\alpha \sin \alpha b & \cos \alpha b
\end{array}\right]\left[\begin{array}{c}
\psi(N b+b) \\
\psi^{\prime}(N b+b)
\end{array}\right]} \\
\text { Here } m_{11}=\cos \alpha b \cos \beta b-\frac{\beta}{\alpha} \sin \alpha b \sin \beta b, m_{12}=-\frac{1}{\beta} \cos \alpha b \sin \beta b-\frac{1}{\alpha} \sin \alpha b \cos \beta b \\
m_{21}=\alpha \sin \alpha b \cos \beta b+\beta \cos \alpha b \sin \beta b, m_{22}=-\frac{\alpha}{\beta} \sin \alpha b \sin \beta b+\cos \alpha b \cos \beta b \\
U_{N}(\chi)=\frac{\sin [(N+1) \arccos \chi]}{\sqrt{1-\chi^{2}}}(\text { defined as chebyshev polynomials ) } \\
\text { and } \chi=\frac{1}{2}\left[\left(\cos \alpha b \cos \beta b-\frac{\beta}{\alpha} \sin \alpha b \sin \beta b\right)+\left(-\frac{\alpha}{\beta} \sin \alpha b \sin \beta b+\cos \alpha b \cos \beta b\right)\right]
\end{gathered}
$$

By substituting both $x=0$ and $x=l=2 N b+b$ into (2) and it's derivative, we have, respectively,

$$
\left\{\begin{array}{l}
\frac{\psi^{\prime}(0)}{\psi(0)}=-\frac{1}{\beta_{0}} \\
\frac{\psi^{\prime}(l)}{\psi(l)}=\frac{A_{N} e^{-\beta_{0} l}}{-\beta_{0} A_{N} e^{-\beta_{0} l}}=\frac{1}{-\beta_{0}}
\end{array}\right.
$$

Thus, the substitution of (7) into (4) produces

$$
\left[\begin{array}{l}
1 \\
\beta_{0}
\end{array}\right]=\left[\begin{array}{cc}
m_{11} U_{N-1}(\chi)-U_{N-2}(\chi) & m_{12} U_{N-1}(\chi) \\
m_{21} U_{N-1}(\chi) & m_{22} U_{N-1}(\chi)-U_{N-2}(\chi)
\end{array}\right]\left[\begin{array}{cc}
\cos \alpha b & -\frac{1}{\alpha} \sin \alpha b \\
\alpha \sin \alpha b & \cos \alpha b
\end{array}\right]\left[\begin{array}{c}
1 \\
-\beta_{0}
\end{array}\right]
$$

Now by multiplying (8) with $\left[\begin{array}{ll}-\beta_{0} A_{1} & A_{1}\end{array}\right]$, and using $\left[\begin{array}{ll}-\beta_{0} A_{1} & A_{1}\end{array}\right]\left[\begin{array}{l}A_{1} \\ \beta_{0} A_{1}\end{array}\right]=0$, we easily obtain

$$
\left[\begin{array}{ll}
-\beta_{0} & 1
\end{array}\right]\left[\begin{array}{cc}
m_{11} U_{N-1}(\chi)-U_{N-2}(\chi) & m_{12} U_{N-1}(\chi) \\
m_{21} U_{N-1}(\chi) & m_{22} U_{N-1}(\chi)-U_{N-2}(\chi)
\end{array}\right]\left[\begin{array}{cc}
\cos \alpha b & -\frac{1}{\alpha} \sin \alpha b \\
\alpha \sin \alpha b & \cos \alpha b
\end{array}\right]\left[\begin{array}{c}
1 \\
-\beta_{0}
\end{array}\right]=0
$$


The expansion of (9) can be changed into

$\left[-\beta_{0} \cos \alpha b\left(m_{11} U_{N-1}(\chi)-U_{N-2}(\chi)\right)-\beta_{0} m_{12} U_{N-1}(\chi) \alpha \sin \alpha b\right]$

$+\left[m_{21} U_{N-1}(\chi) \cos \alpha b+\left(m_{22} U_{N-1}(\chi)-U_{N-2}(\chi)\right) \alpha \sin \alpha b\right]$

$+\left[\left(-\frac{\beta_{0}^{2}}{\alpha} \sin \alpha b\right)\left(m_{11} U_{N-1}(\chi)-U_{N-2}(\chi)\right)+\beta_{0}^{2} m_{12} U_{N-1}(\chi) \alpha \cos \alpha b\right]$

$-\left[m_{21} U_{N-1}(\chi)\left(-\frac{\beta_{0}}{\alpha} \sin \alpha b\right)+\beta_{0}\left(m_{22} U_{N-1}(\chi)-U_{N-2}(\chi)\right) \alpha \cos \alpha b\right]=0$

After the expansion, we have

$$
\begin{aligned}
& \sin (\arccos \chi) \cot (N \arccos \chi)= \\
& =\left(\frac{\beta}{\beta_{0}}-\frac{\beta_{0}}{\beta}\right) \cos \alpha b \sin \beta b+\left(\frac{\alpha}{\beta_{0}}-\frac{\beta_{0}}{\alpha}\right) \sin \alpha b \cos \beta b
\end{aligned}
$$

\section{The Eigen Equation of Electronic Energy in Different Quantum Dot}

\subsection{The Eigen Equation of the Quantum Dot When} $\chi=0$

When $\chi=0$, from (4) and (5), we have, respectively

$$
\begin{aligned}
& \tan \alpha b \tan \beta b=\frac{2 \alpha \beta}{\alpha^{2}+\beta^{2}} \\
& \text { and }\left\{\begin{array}{l}
U_{N-1}(\chi)=0 \\
U_{N-2}(\chi)=1
\end{array}\right.
\end{aligned}
$$

Here $N$ is even number. The substitution of (11) and (12) into (9) produces

$$
\beta_{0} \cos \alpha b-\alpha \sin \alpha b+\frac{\beta_{0}^{2}}{\alpha} \sin \alpha b+\beta_{0} \cos \alpha b=0
$$

The solution of (13) is

$$
\alpha b=n \pi+\arctan \left(\frac{2 \alpha \beta_{0}}{\alpha^{2}-\beta_{0}^{2}}\right)
$$

In order to demonstrates directly the relation of the energy level with the surface potential, by putting $\beta_{0}=\sqrt{\frac{2 m}{\hbar^{2}}\left(U_{1}-E\right)}$ and $\alpha=\sqrt{\frac{2 m}{\hbar^{2}} E}$ into (14), we have

$$
\sqrt{\frac{2 m}{\hbar^{2}} E b}=n \pi+\arctan \frac{2 \sqrt{E\left(U_{1}-E\right)}}{\left(2 E-U_{1}\right)}
$$

When $N$ is uneven number and $\chi=0$, the value of (4) is, respectively,

$$
\left\{\begin{array}{l}
U_{N-1}(\chi)=1 \\
U_{N-2}(\chi)=0
\end{array}\right.
$$

Then, the substitution of $m_{1}, m_{2} m_{3}, m_{4}$ and (17) into Eq.(10) generates

$$
\begin{aligned}
& \beta_{0} \tan ^{3} \alpha b+\frac{\left(\beta^{2}-\beta_{0}^{2}\right) \alpha}{\left(\alpha^{2}+\beta^{2}\right)} \tan ^{2} \alpha b+ \\
& +\beta_{0} \tan \alpha b+\frac{\alpha\left(\beta^{2}-\beta_{0}^{2}\right)}{\left(\alpha^{2}+\beta^{2}\right)}=0
\end{aligned}
$$

By solving (18), we get

$$
\alpha b=n \pi+\arctan \left(\frac{\alpha\left(\beta_{0}^{2}-\beta^{2}\right)}{\beta_{0}\left(\alpha^{2}+\beta^{2}\right)}\right)
$$

Putting $\beta_{0}=\sqrt{\frac{2 m}{\hbar^{2}}\left(U_{1}-E\right)}, \quad \alpha=\sqrt{\frac{2 m}{\hbar^{2}} E}, \quad \beta=\sqrt{\frac{2 m}{\hbar^{2}}\left(E-U_{0}\right)}$ into (16), we also have

$$
\sqrt{\frac{2 m}{\hbar^{2}} E b}=n \pi+\arctan \frac{\sqrt{E}\left(U_{0}+U_{1}-2 E\right)}{\sqrt{\left(U_{1}-E\right)}\left(2 E-U_{0}\right)}
$$

\subsection{The Eigen Equation of the Quantum Dot with $\chi \neq 0$}

When the internal periodic potential is very small, $\Delta$ ( $\Delta=\beta-\alpha$ ) is very small, Thus, $\operatorname{Eq}(6)$ can be simplified as

$$
\begin{aligned}
& \chi=\cos (\alpha b+\beta b)+ \\
& +\left[1-\frac{1}{2}\left(\frac{\beta}{\alpha}+\frac{\alpha}{\beta}\right)\right] \sin \alpha b \sin \beta b \approx \cos (\alpha b+\beta b)
\end{aligned}
$$

By putting $\chi \simeq \cos (\alpha b+\beta b)$ into (11), we have

$$
N(\alpha b+\beta b)=n \pi+\operatorname{arccot}\left(\frac{\alpha}{\beta_{0}}-\frac{\beta_{0}}{\alpha}\right)
$$

Now, we put $\quad \beta_{0}=\sqrt{\frac{2 m}{\hbar^{2}}\left(U_{1}-E\right)}, \quad \alpha=\sqrt{\frac{2 m}{\hbar^{2}} E}$, $\beta=\sqrt{\frac{2 m}{\hbar^{2}}\left(E-U_{0}\right)}$ into (26) again, and have

$$
\left(\sqrt{E}+\sqrt{\left(E-U_{0}\right)}\right) \sqrt{\frac{2 m}{\hbar^{2}}} N b=n \pi+\operatorname{arccot}\left(\frac{\sqrt{E}}{\sqrt{\left(U_{1}-E\right)}}-\frac{\sqrt{\left(U_{1}-E\right)}}{\sqrt{E}}\right)
$$

here $N$ is the total periodic number, $n$ is the quantum number.

Eq.(16), (20)and (23) are defined as the eigen equation of electronic energy in quantum dot, which can clearly describe the relationship of electronic energy with the surface potential, the internal periodic potential and structure parameters. They can more clearly describe the electronic state under different quantum number compared with that 
given only when $\chi>0$ in [17]. Especially, these relationships are simpler and clearer than the eigen equation of quantum dot in [17], which are valuable for the further study of quantum dot.

\section{Conclusions}

This paper demonstrates the matrix method to study the eigen equation of electronic energy in quantum dot. Using Chebyshev polynomials of the second kind, we have deduced three eigen equations, which could clearly describe the relationship of electronic energy with the surface potential, the internal periodic potential and structure parameters. The method used in this paper can also be used to investigate the effect of the surface potential, the internal periodic potential and structure parameters on the electronic energy and electronic level distribution.

\section{REFERENCES}

[1] MJ Steer, DJ Mowbray, WR Tribe, MS Skolnick, MD Sturge, Electronic energy levels and energy relaxation mechanisms in self-organized InAs/GaAs quantum dots, Physical Review B Condensed Matter, 1996, 54(24):17738-17744.

[2] M. H. Abdellatif, J. D. Song, W. J. Choi1, N. K. Cho and J. I. Lee, Quantum dot-like effect in $\mathrm{InGaAs} / \mathrm{GaAs}$ quantum well, Eur. Phys. J. Appl. Phys. 55, 20402 (2011).

[3] T Chen, W Xie, S Liang Optical and electronic properties of a two-dimensional quantum dot with an impurity Journal of Luminescence, 2013, 139(9):64-68.

[4] J R Gell, M B Ward, R J Young, RM Stevenson, P Atkinson, Modulation of single quantum dot energy levels by a surface-acoustic-wave, Applied Physics Letters, 2008, 93(8):081115 - 081115-3,

[5] ZL Yuan, ERAD Foo, JF Ryan, DJ Mowbray, MS Skolnick Many-body effects in carrier capture and energy relaxation in self-organized InAs/GaAs quantum dots, Physica B Condensed Matter, 1999, 272(1-4):12-14.

[6] Yang Xiaojie, Wang Qing, Ma Wenquan, Chen Lianghui. Calculation of energy levels in InGaAs/GaAs quantum dot array. Acta Phys. Sin, 2007, 56(9): 5429).

[7] Tang Nai Yun Chen Xiao Shuang Lu Wei, The effect of size distribution on photoluminescence of excited states from InAs/GaAs quantum dots ACTA Physica Sinica V01. 54, No12, December, 2005, 5855- 5859.

[8] Bianchi Mendez, F Dominguezadame, Enrique Macia. A transfer matrix method for the determination of one-dimensional band structures Journal of Physics A Mathematical and General. 01/1993; 26(1):171-177.

[9] R Kh Gainutdinov, M A Khamadeev, M R Mohebbifar and A A Mutygullina, Self-energy function of quantum-dot states and resonance fluorescence 2014 J. Phys. Conf. Ser. 560 012006

[10] V.I. Boichuk, I.V. Bilynskyi, O.A. Sokolnyk, I.O. Shakleina Effect of quantum dot shape of the GaAs/AlAs heterostructure on interlevel hole light absorption, Condensed. Matter Phys., 2013, vol. 16, No. 3, 33702.

[11] SHAN Yi-hong; FENG Shi-meng; Calculation and correction of nano-level quantum dots Chinese Journal of Quantum Electronics 2014-02 1007-5461.2014.02.018.

[12] Murray, C. B; Kagan, C. R.; Bawendi, M. G. (2000).Synthesis and Characterization of Monodisperse Nanocrystals and Close-Packed Nanocrystal Assemblies". Annual Review of Materials Research 30 (1): 545-610.

[13] Yiming Li, Jinn-Liang Liu, O. Voskoboynikov C. P. Lee, S. M. Sze, Electron energy level calculations for cylindrical narrow gap semiconductor quantum dot, Computer Physics Communications Volume 140, Issue 3, 1 November 2001, 399-404.

[14] Yiming Li, O. Voskoboynikov, C. P. Lee, S.M. Sze, Computer simulation of electron energy level for different shape InAs/GaAs semiconductor quantum dot, Computer Physics Communications, Volume 141, Issue 1, 15 November 2001, 66-72.

[15] Xiao-Yan Gu, Energy for Two-electron Quantum Dots: The Quantization Rule Approach Foundations of Physics Foundations of Physics, December 2006, (36)12, 1884-1892;

[16] K G Dvoyan, E M Kazaryan, L S Petrosyan, Electronic states in quantum dots with ellipsoidal symmetry, Physica E: Low-dimensional Systems and Nanostru..., 2005, 28(4):333-338.

[17] Q.J. Luo, S.M. Feng, L.H. Gu, J.X. Liu, Physica B: Condensed Matter, 481(15) 2016, 137. 